\title{
The JEM-EUSO Global Light System Laser Station Prototype
}

\author{
P. Hunt*, R. Bachman, J. Eser, M. Mantz, L. Wiencke, C. Baron, C. Bigler, D. Bruzgo, \\ M. Burg, A. Cain, A. Cummings, A. Evans, W. Finch, J. Fruit, T. Gallmeyer, C. Geier, \\ J. Gossman, D. Hirsch, R. Hanley, A. Osieczanek, I. Smith, and T. Wills \\ Colorado School of Mines \\ E-mail: phunt@mines.edu
}

\section{for the JEM-EUSO Collaboration}

\begin{abstract}
We describe the design and fabrication of a prototype Global Light System (GLS) laser ground station for the JEM-EUSO project. The GLS will be a network of ground-based UV LEDs and steered lasers to monitor and calibrate the JEM-EUSO cosmic ray detector planned for the International Space Station. The GLS units will generate optical signatures in the atmosphere that are comparable to tracks from cosmic ray extensive air showers (EASs). Unlike the EASs, the number, time, energy, location and direction (for lasers) of GLS events can be specified as JEMEUSO passes $400 \mathrm{~km}$ overhead.

Laser tracks from the GLS prototype will be recorded by prototype detectors in ground-to-ground tests. Distant tracks with low angular speed are of particular interest because these are the types of EAS tracks that will be measured by JEM-EUSO. To do this ground-to-ground tests, the prototype detectors will need to measure the laser through the atmosphere at low elevation viewing angles. The beam energy can be adjusted from 1 to $90 \mathrm{~mJ}$ to compensate for this additional atmospheric attenuation. The frequency tripled YAG laser produces $355 \mathrm{~nm}$ ( $7 \mathrm{~ns}$ pulse) light. This wavelength is near the center of the UV EAS fluorescence spectrum. The system is housed in a utility trailer that can be pulled by a small truck for domestic campaigns or rolled into an industry standard 20 foot container for global deployment. In operation mode, the laser platform inside the trailer is isolated mechanically to maintain beam pointing accuracy. A retractable two stage steering head can point in any direction above the horizon. A slip ring eliminates cable wrap problems. The GLS prototype will be used to test the EUSO-TA detector and will also be used in preflight tests of the EUSO-balloon payload planned for a super pressure balloon mission.
\end{abstract}

The 34th International Cosmic Ray Conference,

30 July - 6 August, 2015

The Hague, The Netherlands

\footnotetext{
* Speaker.
} 


\section{JEM-EUSO and the Global Light System (GLS)}

The Extreme Universe Space Observatory on board the Japanese Experimental Module (JEMEUSO) is an experiment planned for the International Space Station (ISS) to discover the origin of Extreme Energy Cosmic Rays (EECRs). JEM-EUSO will look down at the earth and record the flashes of UV light that are emitted by the Extensive Air Showers (EAS) created when EECRs strike the atmosphere [1]. JEM-EUSO will orbit the earth every 90 minutes at an altitude of approximately $400 \mathrm{~km}$ (Fig. 1). The moving footprint of the detector field of view will be approximately $140,000 \mathrm{~km}^{2}$.

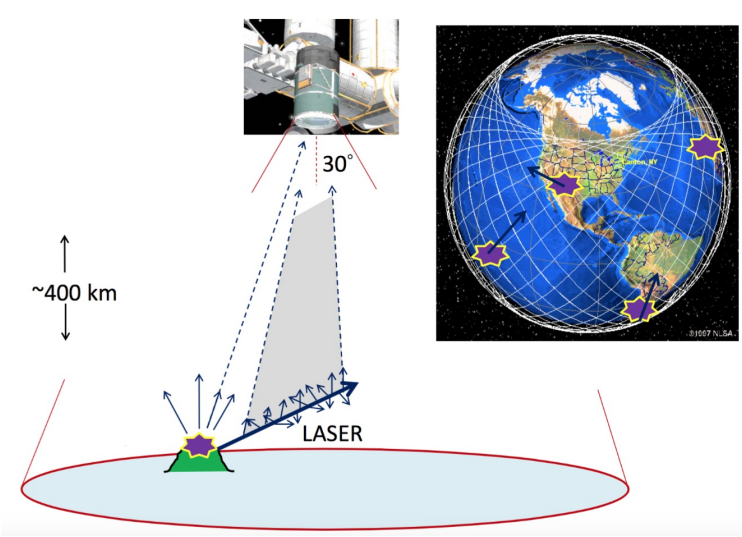

Figure 1: The calibrated lasers of the GLS will generate light tracks that will be measured by JEM-EUSO during its mission [2].

The GLS will generate optical signatures in the atmosphere that will be detected by JEM-EUSO. Directing the scattered light from a pulsed UV laser beam across the field of view of a cosmic ray fluorescence detector is an established technique first used at Fly's Eye [3] and continued at HiRes [4], Telescope Array (TA) [5], and the Pierre Auger Observatory [6]. Data from these programs $[7,8,9,10]$ have demonstrated that the intensity of the light produced from the EASs are comparable to that of the scattered light from lasers propagating through the atmosphere. Showers superimposed on laser light profiles have shown that near vertical $50 \mathrm{EeV}$ showers that are 16 $\mathrm{km}$ from the detector are equivalent to a $5 \mathrm{~mJ}$ laser fired vertically $27 \mathrm{~km}$ from the detector [11]. UV light from the EAS is emitted via excitation of nitrogen in air by the electrons in cosmic ray showers. Light scattering from the laser beam can be described by molecular scattering since the wavelength is much larger than the size of the nitrogen and oxygen molecules.

In contrast to the EASs, the absolute time, energy, and direction can be specified in advance and can be repeated as often as required. Data from the fired shots of the laser can be reconstructed and compared to the known properties of the laser light to monitor the detector performance through out the planned mission.

\section{GLS Prototype}

The GLS Prototype is a portable steered laser system that generates laser tracks having an optical equivalence to EASs of energy $10^{19}-10^{21} \mathrm{eV}$. The prototype is housed and transported in a utility 
trailer. The laser beam can be pointed in any direction above the horizon. For calibration, diagnostics, or transport, the steering assembly can be lowered inside the trailer and a covering hatch closed. The system can be hauled by a pick up truck for national campaigns or can be shipped in a 20 foot shipping container for international campaigns.

\subsection{Laser System}

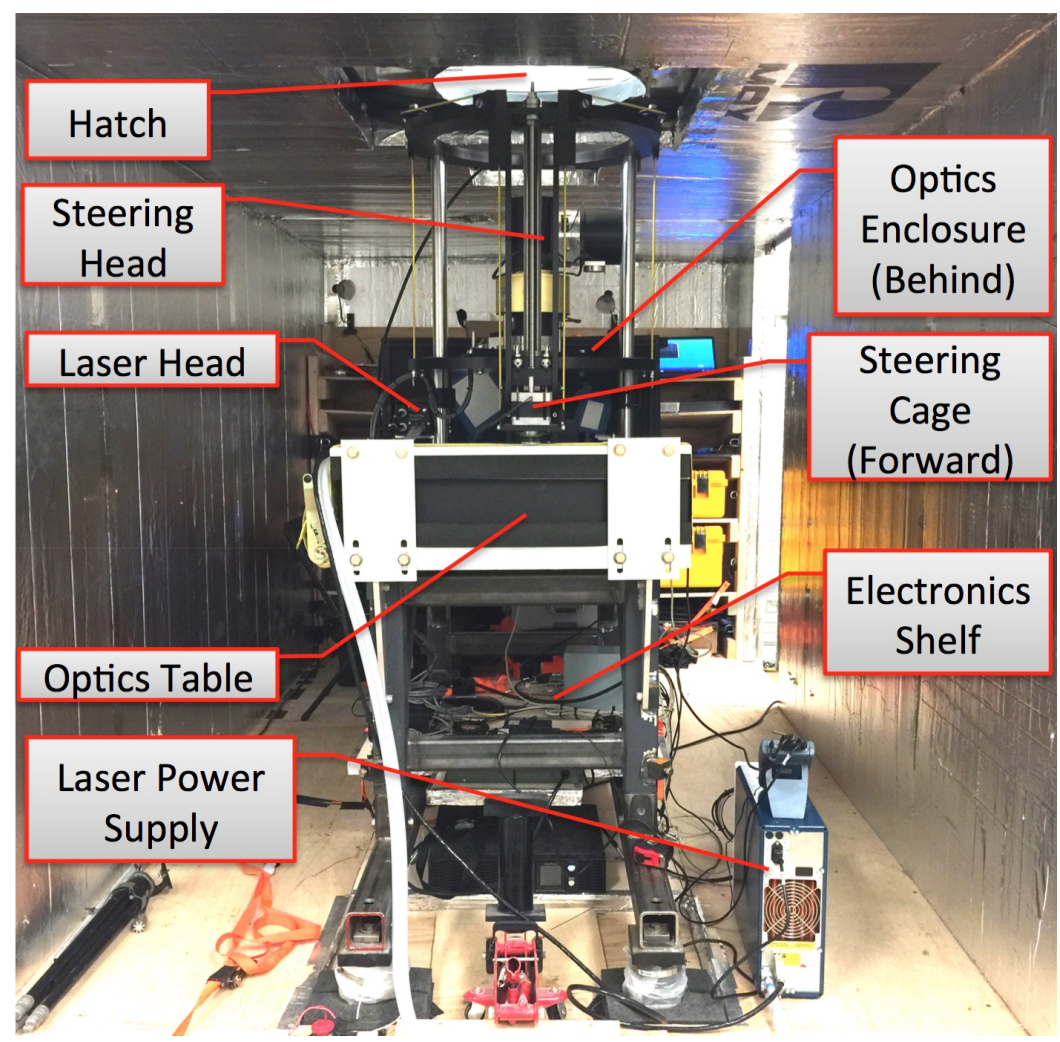

Figure 2: The main components of the laser system of the GLS Prototype.

The system (Fig. 2) features a frequency tripled, pulsed, Nd:YAG, 90 mJ CFR400 laser from Quantel Laser [12]. The laser wavelength of $355 \mathrm{~nm}$ falls near the middle of the nitrogen fluorescence spectrum [13]. An internal attenuator provides a range of beam energies that span two orders of magnitude. The optical setup also includes dichroic and dual band mirrors, a quarter waveplate, two energy probes with radiometers, and a custom GPS clock [14]. The laser and radiometers communicate with a custom single-board computer, which can set the laser attenuation, specify and record absolute time the laser is fired, and record the energy of every laser pulse. The beam steering system uses a periscope style design with two orthogonal rotary stages for independent control of the azimuth and elevation pointing angles. The latter is connected through a slip ring to eliminate cable wrap problems and elevating platform that supports the two-stage steering head (Fig. 3).

Energy probe 1 measures $4 \%$ of the beam energy of every pulse via a UV reflecting window (Fig. 4). The full beam energy can be measured downstream of all of the optics by rotating the steering head to point down at a calibration energy probe (probe 2) that is mounted on the optical table. 

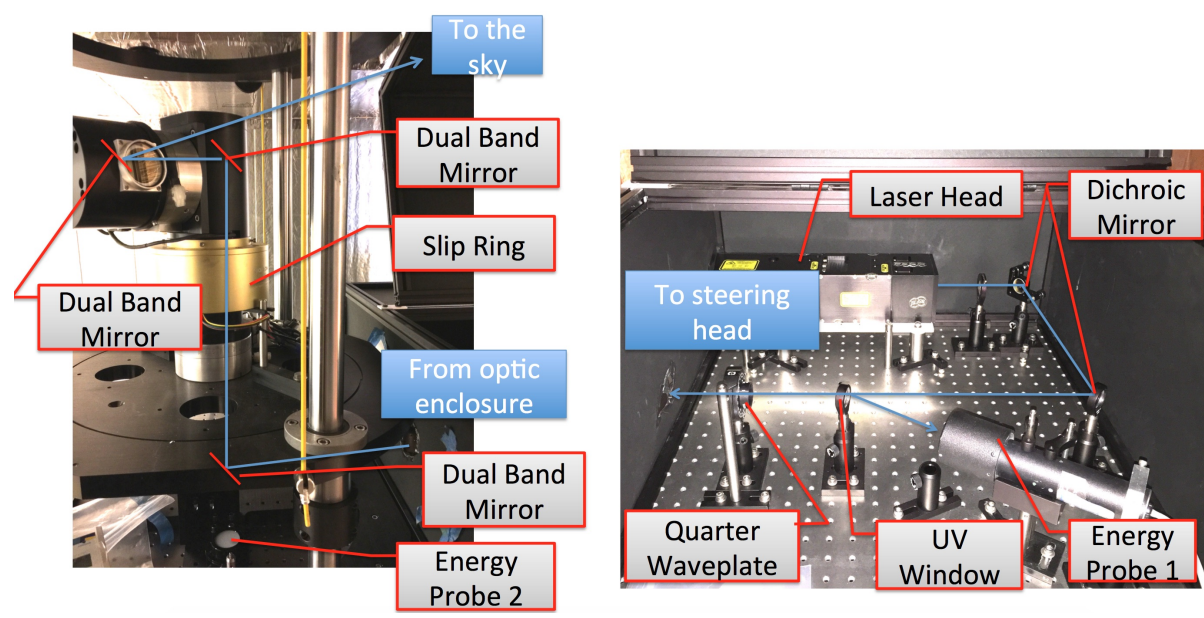

Figure 3: Laser propagation starting from the laser head in the optics enclosure (right) traveling through and out of the steering head to the sky (left).

The ratio of probe 2 to probe 1 yields a calibration factor (CF). During normal operation, the measurements of probe 1 are multiplied by the CF to yield the beam energy sent to the sky (Figs. $4 \& 5)$.

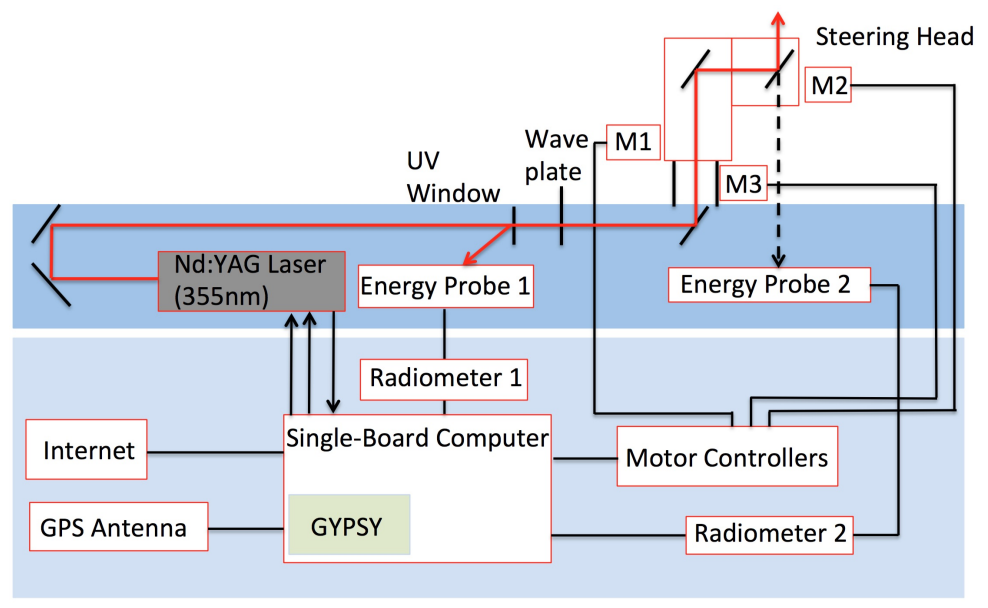

Figure 4: Block diagram of the GLS Prototype laser system.

The quarter waveplate is used to circularly polarize the vertically polarized light that is emitted from the laser. To achieve circular polarization, the quarter waveplate is rotated in increments while the energy is measured through a rotating polarizing cube until the fluctuations in measured energy are minimized over a full rotation of the cube (Table $1 \&$ Fig. 6). The quarter waveplate can be exchanged with a depolarizer to randomize the net polarization to less than $5 \%$, or a half waveplate which can rotate the linear polarization to a desired orientation.

Three stepper motors control the motion of the periscope beam steering assembly. Two of the motors rotate the orthogonal steering stages. The third motor lifts the steering head through the opened hatch. Each stepper motor is accurate to one step which corresponds to $0.0125^{\circ}$ for beam steering. For each rotation stage (azimuth and elevation angles), a dual band (355 $\mathrm{nm}$ and 632 

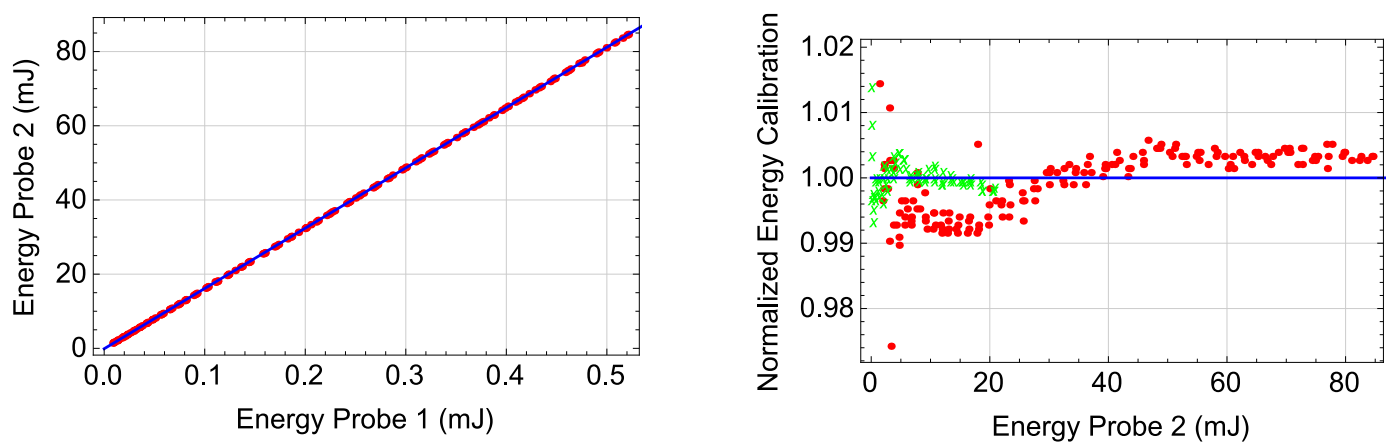

Figure 5: Energy Calibration of the laser (left). Variations of the CF from $0.40 \mathrm{~mJ}$ to $85 \mathrm{~mJ}$ is less than $1 \%$ (right). Energy measured with (green $\mathrm{x}$ ) and without (red dot) a 10\% filter in front of probe 1 to prevent damage to the probe during high energy measurements.

Table 1: Parameters and Specifications of the GLS Prototype

\begin{tabular}{|l|c|l|c|}
\hline Wavelength & $355 \mathrm{~nm}$ & Energy Calib. (Rel.) & $<2 \%$ \\
\hline Energy/Pulse & $0.40-90 \mathrm{~mJ}$ & Energy Calib. (Abs.) & $<5 \%$ \\
\hline Pulse Width & $7 \mathrm{~ns}$ & Power Draw (Laser firing) & $225.8 \mathrm{~W}$ \\
\hline Max. Repetition & $20 \mathrm{~Hz}$ & Steering Precision (Rel.) & $0.0125^{\circ}$ \\
\hline Energy Stability (Spec) & $<2 \%$ & Steering Precision (Abs.) & $<0.20^{\circ}$ \\
\hline Polarization & $\pm 5 \%$ (circ.) & Optics Table Level & $<0.02^{\circ}$ \\
\hline Absolute Timing & $\pm 20 \mathrm{~ns}$ & & \\
\hline
\end{tabular}
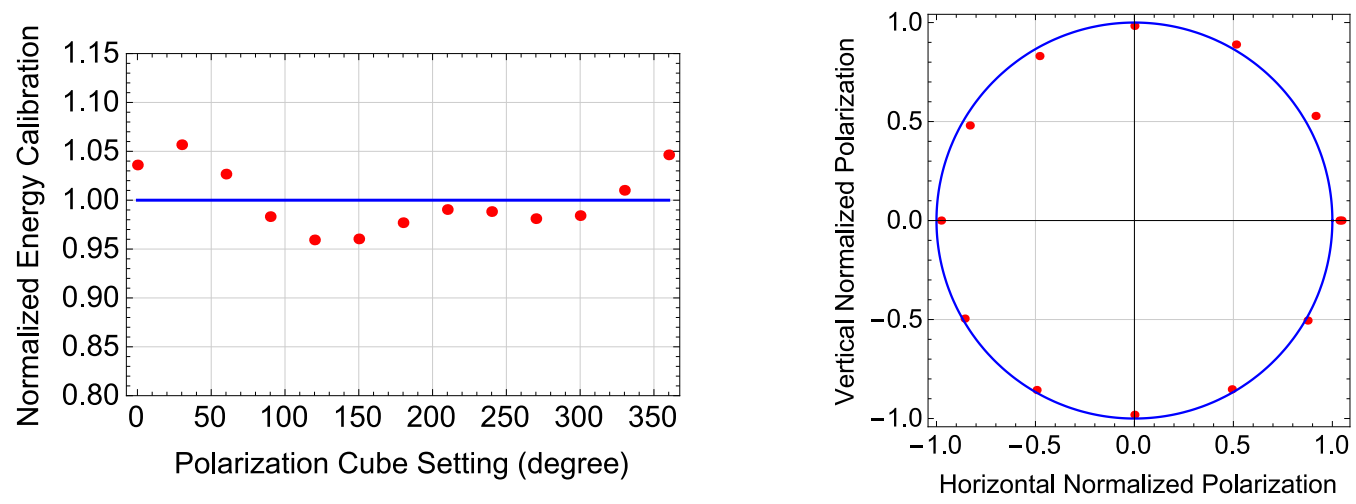

Figure 6: An example of a polarization calibration. Polarization of the laser light is shown to be within $5 \%$ of circular polarization (left) and represented in a polar plot (right).

$\mathrm{nm})$ mirror installed on a 3D axis mount provides a constant reflection angle of $45^{\circ}$. To verify that the mirrors are truly at $45^{\circ}$, an alignment process (Fig. 7) rotates each stage by $180^{\circ}$ and requires that the beam spots before and after the rotations are aligned with the targets of a custom laser alignment plate (Fig. 7). For this test, a $635 \mathrm{~nm}$, self-leveling, He-Ne laser can be used in place of the $90 \mathrm{~mJ}$ pulsed laser. By precisely leveling the optical table (Section 2.2), adjusting the dual band mirrors in the steering head and the dichroic mirrors in the enclosure, the beam pointed into the sky with an accuracy is currently better than $0.2^{\circ}$. 

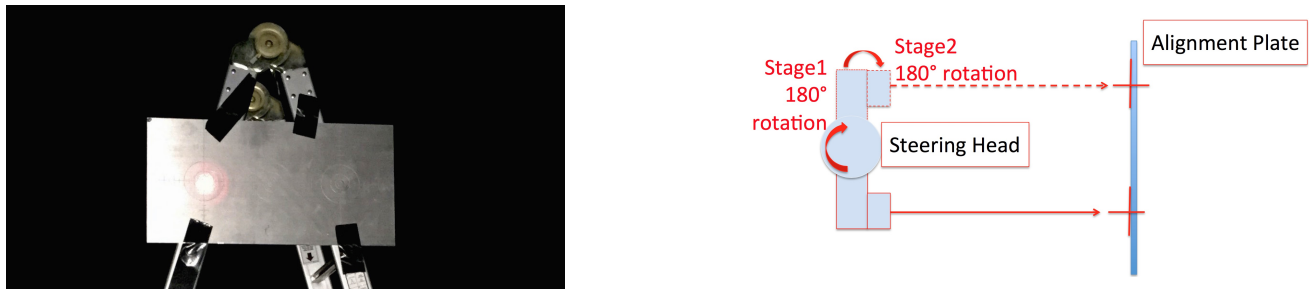

Figure 7: Custom alignment plate (left) used to check alignment of steering head. Two targets are spaced apart to match horizontal displacement of the beam when the 2 stages are rotated by $180^{\circ}$ (right).

\subsection{Housing \& Infrastructure}

The laser system is housed in a utility trailer that has been modified with a sliding hatch so that the raised steering head can be pointed in any direction above the horizon (Fig. 8). The system can be transformed between Transport and Operation Mode. In Transport Mode, the optical table frame is secured to the trailer frame through a redundant arrangement of bolts and jacks. Neoprene blocks are secured between the optics table and the table frame to dampen vibration.
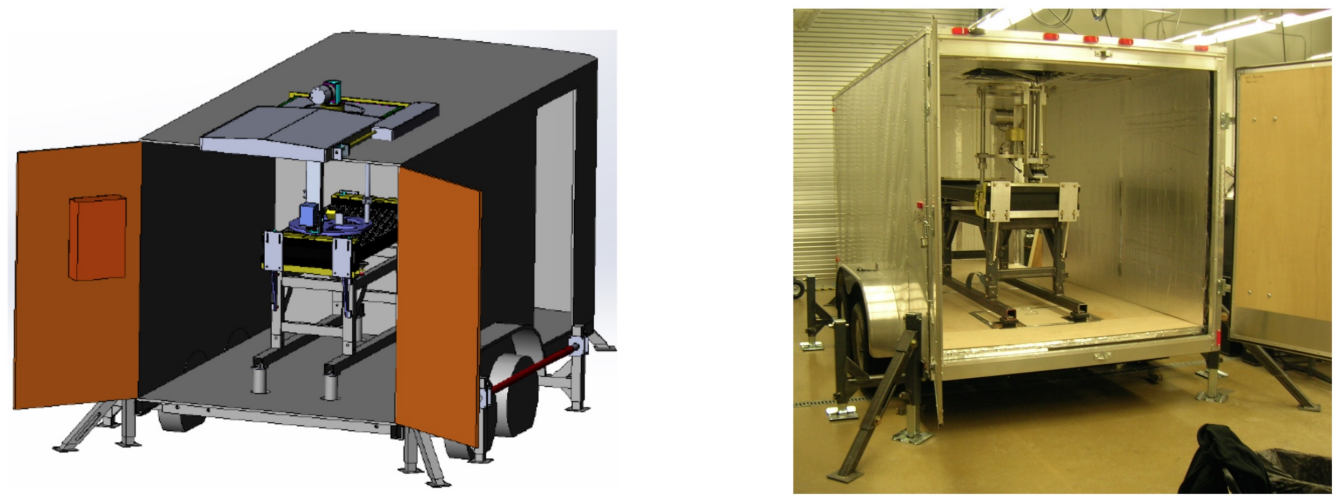

Figure 8: 3D Model of the GLS Prototype (left) and the fabricated GLS Prototype (right).

When the trailer reaches its destination, the system is transformed into Operation Mode (Fig. 8). A jack assembly is installed on each corner of the trailer for leveling and stabilization. The optics table and frame are unbolted from the trailer frame and are supported on separate outrigger jack assemblies. The outrigger jacks serve both to level the laser optical table and steering assembly and to mechanically isolate these components from the trailer. The configuration allows the trailer to be shaken by wind outside or by people walking inside without affecting the beam direction.

\subsection{First Light Campaigns}

The GLS Prototype has provided the first laser tracks to test the EUSO-TA detector at the Telescope Array near Delta, Utah [15]. Tracks from the GLS Prototype have been detected at approximately $34 \mathrm{~km}, 40 \mathrm{~km}, 60 \mathrm{~km}$, and $100 \mathrm{~km}$ from the EUSO-TA detector (Fig. 9) - March 13-14, 2015 and May 20-24, 2015. The $34 \mathrm{~km}, 60 \mathrm{~km}$, and $100 \mathrm{~km}$ sites lie on the intersection between dirt roads and a straight line projected from EUSO-TA through the Central Laser Facility (CLF). 


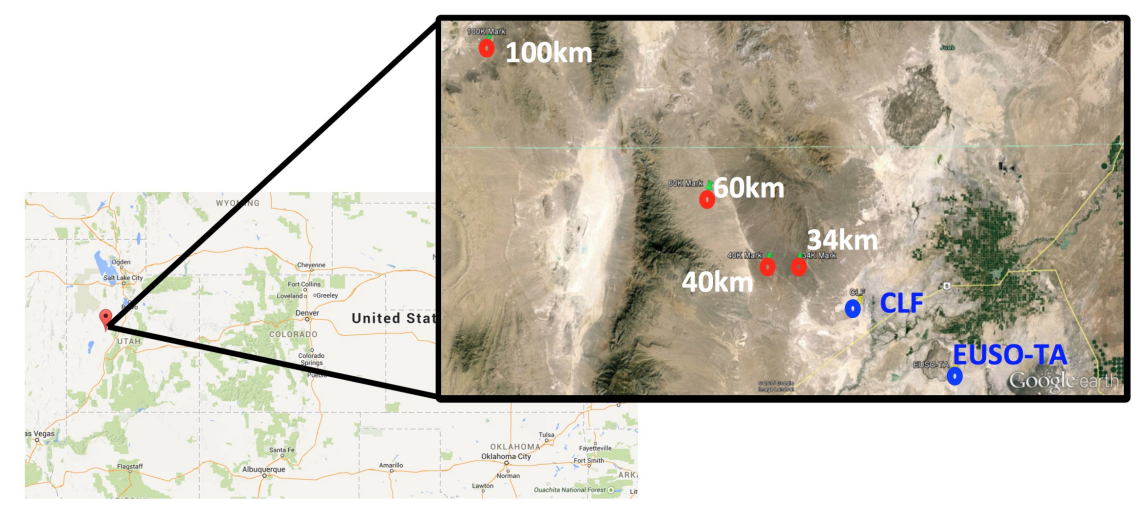

Figure 9: Locations of test sites and CLF/EUSO-TA

A laser track from the GLS Prototype recorded during the May 2015 campaign is shown in Fig. 10. The image is the superposition of 150 frames. Noise has been removed from the sequence to show the laser as it propagates through the atmosphere. For each location, the goal is to record a series of optical tracks at variable laser attenuations and elevation angles at various distances that will be compared to simulated data to test the performance of the EUSO-TA instrument.

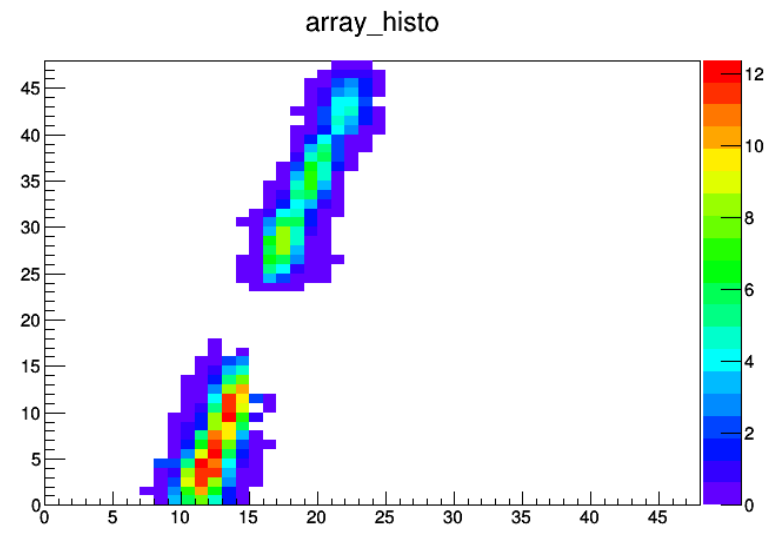

Figure 10: Laser track captured by EUSO-TA during the May 2015 campaign (the break in track is likely due to a broken detection unit). Each pixel corresponds to a $0.25 \times 0.25$ degree field of view and the color scale is a relative intensity of measured light from EUSO-TA. The laser system is located slightly below the track.

\section{Conclusions}

The Global Light System is designed to provide calibrated optical flashes and tracks to monitor the performance of JEM-EUSO during its mission. Drawing on experience with systems at HiRes and the Pierre Auger Observatory, the design and fabrication of the GLS laser station prototype has been completed. The system is being used to characterize the performance of EUSO-TA. First light campaigns have established a new record $(100 \mathrm{~km})$ in laser-detector separation. The test procedures 
being developed with EUSO-TA will be applied to the EUSO-Balloon instrument prior to a long duration stratospheric flight planned for 2017 [16].

Acknowledgment: This work is supported by NASA grants NNX13AH55G, NNX13AH53G.

\section{References}

[1] P. Picozza et al. (JEM-EUSO Collaboration), The JEM-EUSO Program in Proc. 34th ICRC, (The Hague), (2015).

[2] L. Wiencke et al. (JEM-EUSO Collaboration), The JEM-EUSO Global Light System in Proc. 33rd ICRC, (Rio de Janeiro), (2013).

[3] Fly's Eye Collaboration, R.M. Baltrusaitis et al., The Utah Fly's Eye Detector, Nucl. Instrum. Meth. A 240, 410 (1985).

[4] The HiRes Collaboration, T. Abu-Zayyad et al., The Prototype High-Resolution Fly's Eye Cosmic Ray Detector, Nucl. Instrum. Meth. A 450, 253 (2000).

[5] H. Kawai et al., Telescope Array experiment, Nuclear Physics B (Proc. Suppl.) 175-176, 221-226 (2008).

[6] Pierre Auger Collaboration, J. Abraham et al., The Fluorescence Detector of the Pierre Auger Observatory, Nucl. Instrum. Meth. A 620, 227 (2010) [arXiv:0907.4282 [astro-ph.IM]].

[7] Bird, D.J. et al., Detection of a Cosmic Ray with Measured Energy Well Beyond the Expected Spectral Cutoff Due to Cosmic Radiation, Ap. J., 441, 144 (1995).

[8] C. Cannon, L. Pedersen, R. Riehle, J. Thomas and L. Wiencke (HiRes Collaboration), Probing the HiRes Aperture Near $10^{20} \mathrm{eV}$ With a Distant Laser in Proc. 28th ICRC, (Tsukuba), (2003).

[9] Pierre Auger Collaboration, P. Abreu et al., The Exposure of the Hybrid Detector of the Pierre Auger Observatory, Astropart. Phys. 34, 368 (2011) [arXiv:1010.6162 [astro-ph.HE]].

[10] Pierre Auger Collaboration, P. Abreu et al., Techniques for Measuring Aerosols using the Central Laser Facility at the Pierre Auger Observatory, JINST 8, p04009 (2013) [arXiv:1303.5576].

[11] Pierre Auger Collaboration, P. Abreu et al., Techniques for Measuring Aerosol Attenuation using the Central Laser Facility at the Pierre Auger Observatory, JINST 8, p04009 (2013) [arXiv: 13035576v].

[12] www.quantel-laser.com.

[13] AIRFLY Collaboration, M. Ave et al., Precise Measurement of the Absolute Fluorescence Yield of the 337 nm Band in Atmospheric Gases, Astropart. Phys. 28, 41 (2007).

[14] J.D. Smith, et al. (Pierre Auger Collaboration), GPSY 2: A Programmable Hardware Module for Precise Absolute Time Event Generation and Measurement in Proc. 30th ICRC, (Rome), (2007).

[15] M. Casolino et al. (JEM-EUSO Collaboration), ESUO-TA, a ground precursor to JEM-EUSO telescope on the Telescope Array site: status and updates in Proc. 34th ICRC, (The Hague), (2015).

[16] P. von Ballmoos et al. (JEM-EUSO Collaboration), The EUSO-Balloon mission in Proc. 34th ICRC, (The Hague), (2015). 